\title{
SCIENCE
}

NEW YORK, MAY 6, 1892.

\section{PRELIMINARY NOTE ON THE RELATIONS OF THE MOTOR MUSCLES OF THE EYES TO CERTAIN FACIAL EXPRESSIONS.}

In the course of some years of close observation of the anomalies of the muscles which govern the movements of the eyes, the fact that remarkable changes often follow the modification of the conditions of these muscles led me not only to regard with greater care these facial changes, but to bring to the subject the aid of photography, by which means only these expressions could be accurately registered. Photographic portraits giving a direct front-view of more than two thousand persons have thus been made. In each case a record, as full and as accurate as I have been able to obtain, of the state of these eye muscles has been made, and in the majority of the cases careful observations have been repeated many times during some weeks or months.

We have thus, for the first time, a series of observations in which the facial expressions are registered by photography, while painstaking determinations of the ocular-muscle conditions are faithfully recorded.

Beyond this, in a great number of instances, photographs have been taken at various stages of modification of these muscles, thus affording a comparative study of the face under varying conditions of the eye muscles.

The result of this study has been to demonstrate that certain well-defined types of facial expression are not only associated with but are dependent upon certain relative tensions of the oculo-motor muscles.

An elementary knowledge of the conditions of these muscles, as commonly presenting themselves in practice, is necessary to an understanding of what follows.

In an ideal condition of the eye muscles, they should, when the minimum of effort of all the opposing forces is exercised, so adjust the eyes that the lines of vision will be practically parallel. More exactly, the adjustments should be such that the visual lines would meet only at a point situated at a very considerable distance in front of the eyes.

This condition of perfect equilibrium of all the opposing eye-muscles does not always exist. Indeed, such a condition is the exception. (In this statement no reference to the conditions known as strabismus or squint is intended; in fact, in this connection all such exaggerated anomalies are left absolutely out of consideration, the purpose being to consider only the conditions in which single vision with the two eyes is maintained).

The condition of equilibrium above described is known as orthophoria.

In case of a normal excess of tension on the part of the muscles which rotate the eyes inward, the visual lines, should no restraining force be brought into action, would drift toward each other and visual conf usion would result: In that case, by means of a voluntary but unconscious effort on the part of the opposing muscles, the lines of vision might be propertyladjusted. This condition, in which there is a nor mal tendency of the visual lines to approach, but in which there is ability to restrain that tendency, is known as esophoria.

The opposite condition, in which the normal tension of the muscles which rotate the eyes outward predominates and which, if unrestrained, would cause the visual lines to diverge, is known as exophoria,

Another condition, in which a tendency of one of the visual lines to rise above its fellow is found, is known as hyperphoria.

Compound conditions called hyperesophoria and hyperexophoria are also found. The terms suggest the elements of these conditions.

In the great majority of persons, some one of these socalled anomalies is to be found. While the condition of absolute orthophoria is, perhaps, not to be expected, that of a near approach to it is sometimes, although exceptionally, found.

The conditions of esophoria and of exophoria are much more common. Hyperphoria would appear to be somewhat less common than the last two.

With each of these conditions of relative tensions of the eye muscles is commonly associated a type of expression

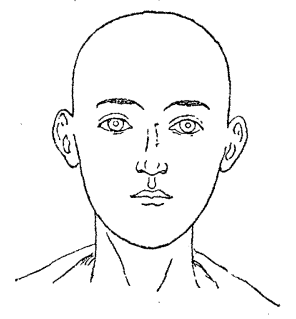

Fra. 1.

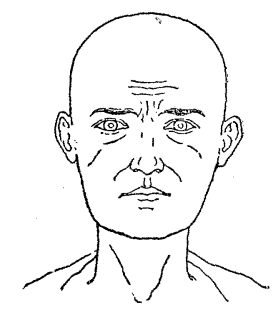

FrG. 2. sometimes slightly but often extremely well marked. That there are found apparent exceptions to this rule, as to most other rules, is true. Yet the law is so generally prevalent as to enable us to classify nearly all faces by its assistance.

The great elasticity of muscles, together with the peculiar characteristics of the sub-dermal tissues in childhood and early youth, and the loose and wrinkled character of the skin in advanced age, to a greater or less degree disguise the characteristic expressions arising from these various muscular tensions. The types are therefore most conspicuous in early adult and in middle age.

In the accompanying diagrams, I have endeavored to illustrate the peculiarities of four principal types of facial expression as governed by the eye muscles.

With the state of equilibrium of these muscles, orthophoria (Fig. 1), the expression is one of greater repose than with any of the other states of the eye muscles. The eyebrows, which constitute one of the most striking of all the facial features, form each a moderate and regular curve, marking the border of the orbit, the lower border of the brow corresponding to the orbital border. The inner extremity descends towards the nose, but does not turn downward into the depression bounded by the nose and the orbit. There is 
no sharp turn or sudden increase of curve at either extremity. The mouth is nearly horizontal or curving very slightly upwards at the centre. The lips in repose are firm but not compressed, and the upper one is well proportioned. The chin is rounded, neither square nor pointedly oval. The lines of the forehead are not usually conspicuous. The nasolabial lines curve outward beyond the angles of the mouth, less horizontally than with esophoria, and less vertically than with exophoria. The curved line below the lower lid is nearly in exact conformity with the curve made by the fold of the upper eye-lid when it is moderately raised.

The absence of special tensions of the facial muscles, in this well-balanced face, permits a quick and easy play of the features, and the habitual absence of any forcible regulation of the eyes or of the face is conducive to a mental equilibrium and to physical endurance.

With esophoria (see Fig. 2) the brows are compressed, often flattened. The extremities often curve suddenly downwards, the inner extremity sinking into the depression bounded by the nose and orbital border. The eyelids are, in a considerable proportion of cases, not as fully opened as in orthophoria, and much less separated than in the typical cases of exophoria. Two rather strong vertical lines making each an angle with the inner extremity of a brow extend apward nearly parallel, upon the forehead, as shown in the

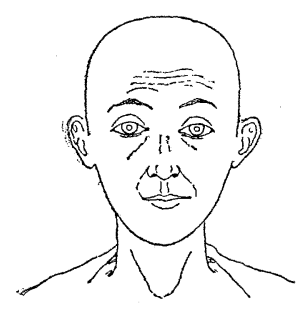

FIG 3.

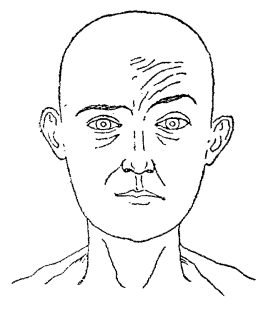

FiG. 4. diagram. The upper lip is usually short, and the curve of the centre of the mouth upward is often pronounced. The lips are firmly compressed in repose, but in young persons with deficient physical force, the lips may be habitually open. The ehin is broad and the naso-labial lines make a wider excursion outward than in orthophoria. The expression in moderate esophoria suggests firmness of character and resolute purpose.

With exophoria (see Fig. 3) the brows are usually strongly arched, often drawn upward upon the forehead, the inner extremity being often removed above and away from the nose. The lines running upward from the side of the nose, when present, are likely to diverge as they ascend. The transverse lines of the forehead are often conspicuous and are higher on the forehead than those which occur with esophoria. The upper lip is long, the centre of the mouth curves downward and the chin is pointedly oval. The facial lines are more vertical than with either of the conditions described, giving to the face the suggestion of length. The lips are not compressed and are liable to be loose and slightly open.

The expression of exophoria suggests more of idealism than of determinate purpose.

Hyperphoria is characterized by irregular features (Fig. 4). The tendency of one visual line to rise above the other demands a restraining effort in which the facial muscles of ten take an important part. On the side, the visual line of which tends to rise above the other, the brow is depressed, while the brow of the side whose visual line tends downward is elevated. Thus the brow and neighboring tissues of one side aid in depressing the front of the eye, while, on the other hand, by the elevation of the opposite brow less demand is made upon the muscle which is required to rotate its eye upward.

These contrary actions demanded by the relations of the visual lines in hyperphoria affect the whole face, resulting in a want of harmony of the two sides. The angle of the mouth, on the side on which the brow is depressed, is drawn upward, while the other angle is depressed. Thus one side of the face is longer than the other. On one side, the lines of esophoria are found, and on the other, those of exophoria.

In the sketch here given no attempt has been made to describe all the various gradations between these types nor to explain the exceptions. It has been the purpose of this communication only to present the general characteristics of some of the most typical forms of expression which have their origin in the efforts to adjust the eyes.

New York.

George T. Stevhins, M.D.

\section{THE ARCHITECTURAL EXHIBITION IN BROOKLYN.}

THERE is no question but that we stand upon the verge of a great popular revival of interest in architecture. Architectural books and magazines command wide circulations and numerous purchasers. Vast sums of money are yearly expended in building-call it architecture if you will. Exhibitions of architectural drawings have become regular features of winter life in the larger cities. On all hands greater interest is being manifested in the art than ten or twenty years ago seemed possible.

To Brooklyn belongs the credit of having supported the only popular organization for the study of architecture in the country. Professional and student bodies perform a very different function than that which naturally belongs to an institution resting upon non-professional and popular bases. The Brooklyn Institute of Arts and Sciences, especially under the direction of its present efficient head, Prof. Franklin W. Hooper, has achieved a national reputation. An organization that maintains forty-five distinct courses of public lectures, numbering on an average 400 annually, must be reckoned among the most powerful intellectual forces in the country, a power which is not limited by the fact that it confines its operations to the city of Brooklyn.

It is quite in keeping with the progressive policy of the Institute that it should provide the citizens of its native city with an exhibition of architectural drawings, which is noteworthy not only as an evidence of progress on the Institute's part, but as being the most ambitious attempt of the kind yet made in Brooklyn. First attempts are always liable to leave something to be desired, but the Brooklyn Exhibition, which has just closed, was so good on the whole that little fault may be found with it. On the contrary the gentlemen having the matter in charge are to be thoroughly congratulated on succeeding so well. Whatever faults may be found with the exhibition are faults inherent in all architectural exhibitions as a class, and are by no means limited to Brooklyn alone.

And this chief fault is the matter of the exhibition. There is no important subject on which such erroneous views prevail as on architecture. This is a fact that requires no argument. It follows as a natural consequence, therefore, that every time the architects attempt to initiate the public 